\title{
Invaginación gastroduodenal secundaria a tumor estromal gastrointestinal
}

\section{Gastroduodenal intussusception secondary to gastrointestinal stromal tumor}

\author{
Julián Herrera ${ }^{1}$, Juliana L. Molina ${ }^{2 *}$ y Erika Osorio ${ }^{2}$
}

${ }^{1}$ Departamento de Imágenes diagnósticas, Hospital Manuel Uribe Ángel, Envigado; ${ }^{2}$ Departamento de Cirugía, Universidad de Antioquia, Medellín. Colombia

\section{Resumen}

La invaginación del tracto gastrointestinal es infrecuente en la población adulta, más aún aquella del segmento gastroduodenal. A diferencia de la población pediátrica, la mayoría de los casos de invaginación tienen una causa secundaria identificable, siendo los tumores los más frecuentes. Presentamos el caso de una mujer de 49 años con síntomas crónicos e inespecíficos, en quien se diagnostica por medio de una tomografía computarizada contrastada de abdomen una masa gástrica que genera invaginación gastroduodenal secundaria, tumor que posteriormente se identifica en la patología como un tumor estromal gastrointestinal.

Palabras clave: Intususcepción. Neoplasias. Tumor estromal gastrointestinal. Dolor abdominal.

\section{Abstract}

Gastrointestinal intussusception is an infrequent entity in adult patients. Even rarest among this are cases involving the gastroduodenal segment. In contradistinction to the pediatric population, most of cases in adult patients have an identifiable cause, tumors being the main etiology. In this article we present the case of a 49 year old woman with chronic and nonspecific symptoms, in whom a gastric mass that generates secondary gastroduodenal intussusception was diagnosed by a contrast computed tomography of the abdomen, a tumor that is later identified in the pathology as a gastrointestinal stromal tumor (GIST).

Key words: Intussusception. Neoplasms. Gastrointestinal stromal tumors. Abdominal pain.

\section{Introducción}

La invaginación se define como el telescopaje de un segmento proximal del intestino a uno adyacente distal. Es una causa relativamente común de obstrucción intestinal en la población pediátrica a diferencia de los adultos, quienes representan $5-10 \%$ de las invaginaciones y solo el $10 \%$ de estas compromete el segmento gastroduodenal. En el $90 \%$ de los casos existe una patología que predispone a la invaginación en la población adulta, siendo los tumores los principales responsables ${ }^{1}$.
Disponible en internet: 21-09-2021 Rev Chil Radiol. 2021;27(3):151-153 www. resochradi.com (https://creativecommons.org/licenses/by-nc-nd/4.0/). 
El tumor estromal gastrointestinal (GIST) representa del 1 al $3 \%$ de todas las neoplasias gastrointestinales. Son tumores mesenquimales con potencial maligno que se originan de la transformación neoplásica de las células intersticiales de Cajal, pueden encontrarse en cualquier localización del tracto gastrointestinal, siendo más común su presentación en el estómago e intestino delgado. Su crecimiento predominante exofítico los hace una causa infrecuente de invaginación ${ }^{2,3}$.

\section{Descripción del caso}

Mujer de 49 años sin antecedentes relevantes, quien consulta por cuadro clínico de cuatro meses de evolución de dolor abdominal intermitente e inapetencia y tres días de melena, sin síntomas obstructivos asociados. Al examen físico se presenta con estabilidad hemodinámica, abdomen doloroso a la palpación en epigastrio, sin signos de irritación peritoneal ni masas palpables.

Se realizó tomografía computarizada contrastada de abdomen con hallazgo de masa gástrica bien definida, de morfología ovalada, dependiente del fondo gástrico, de aproximadamente $45 \times 40 \mathrm{~mm}$. La lesión está desplazada en sentido distal, causando invaginación gastroduodenal secundaria. No se encontraron signos de compromiso extramural o metastásico (Fig.1). El estudio endoscópico complementario confirma una lesión de gran tamaño en el fondo gástrico de aspecto polipoideo pediculado con zonas de necrosis en su superficie apical, sugiriendo la posibilidad de GIST de aproximadamente $5 \mathrm{~cm}$ de diámetro, la cual tiende a desplazarse a través del canal pilórico hacia el duodeno. La paciente es sometida a gastrectomía subtotal con linfadenectomía radical abierta. El estudio histopatológico confirma tumor del estroma gastrointestinal (Fig. 2).

\section{Discusión}

La invaginación del tracto gastrointestinal es una patología infrecuente en la población adulta. Del total de casos, el $90 \%$ son intestinales y el $10 \%$ restantes son gastroduodenales, también conocido como síndrome de ball valve 3,4 .

El $70 \%$ de los GIST se ubican en el estómago, seguido por el intestino delgado y menos frecuente en colon. Su incidencia es igual para ambos sexos y es más frecuente en mayores de 40 años. La presentación clínica es inespecífica, usualmente consiste en hemorragia del tracto digestivo evidenciado como melena, hematemesis o anemia, dolor abdominal, obstrucción intestinal y otras complicaciones (Tabla 1);

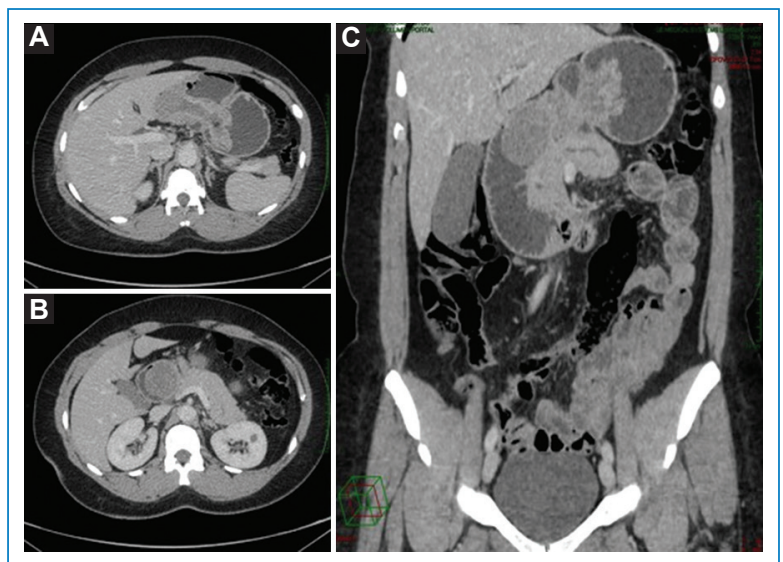

Figura 1. Mujer de 49 años con invaginación gastroduodenal. Tomografía computarizada de abdomen con contraste intravenoso. A y B: cortes axiales donde se evidencia segmento gástrico en duodeno, no hay signos de perforación ni compromiso isquémico. C: corte coronal que muestra masa gástrica bien definida, dependiente del fundus gástrico, de aproximadamente $45 \times 40 \mathrm{~mm}$ invaginada con dilatación de cámara gástrica y duodeno.

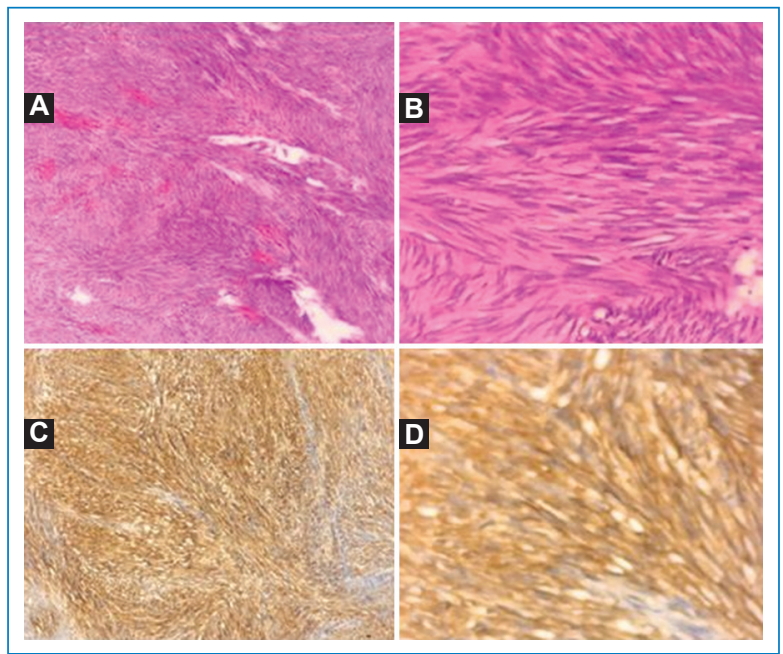

Figura 2. A y B: cortes de histopatología. Lesión neoplásica con patrón fusocelular, tinción hematoxilinaeosina (10X-40X). C y D: lesión neoplásica positiva para KIT (10X-40X).

hasta en un tercio de los pacientes el diagnóstico es incidental $^{5}$. La invaginación gastroduodenal es una presentación muy infrecuente con pocos reportes de casos publicados ${ }^{6,7}$. Al igual que en el caso reportado por Castro, et al., el dolor abdominal y las alteraciones en relación con la alimentación fueron las principales molestias en la paciente ${ }^{8}$.

Ssentongo, et al., realizaron una revisión de los casos de invaginación secundaria a GIST publicados 
Tabla 1. Complicaciones más frecuentes de los tumores estromales gastrointestinales

\begin{tabular}{|l|c|}
\hline Presentación clínica & $\%$ de pacientes \\
\hline Sangrado gastrointestinal & 48,9 \\
\hline Obstrucción intestinal: & 28,3 \\
- Obstrucción de la luz & 54 \\
\hline Intususcepción & 46 \\
\hline Sangrado intraperitoneal & 15,2 \\
\hline Rotura/peritonitis & 7,6 \\
\hline
\end{tabular}

Adaptada de Sorour, et al., $2014^{5}$

desde 1983 al 2018, incluyendo 18 casos con una media de 60 años, con predominio femenino. En el $22 \%$ de los casos los tumores se localizaban en el estómago, el $17 \%$ en yeyuno y el $6 \%$ en duodeno. Diez de los casos tenían invaginación gastroduodenal ${ }^{2}$.

Para el diagnóstico definitivo es necesario el estudio histopatológico de la lesión, sin embargo, algunos hallazgos en la tomografía son característicos del GIST: estos tumores aparecen como masas de densidad de partes blandas que nacen de la pared del tracto gastrointestinal y pueden protruir hacia el lumen o presentar un comportamiento predominantemente exofítico. Su tamaño es muy variable, desde centímetros a voluminosas masas. Suelen ser hipervasculares, con un realce heterogéneo con el contraste endovenoso por la presencia de necrosis central o hemorragias ${ }^{9,10}$; por esto la tomografía es la imagen de elección. Otras patologías que pueden presentar hallazgos imagenológicos y clínicos similares, principalmente tumores mesenquimatosos como los de músculo liso, leiomioma o leiomiosarcoma, tumores neurogénicos, lipomas, liposarcomas, tumor carcinoide y fibromas, entre otros ${ }^{11,12}$.

El manejo definitivo es la resección quirúrgica abierta - laparoscópica con mejores resultados en cuanto a morbilidad de esta última. Según lo reportado en la literatura, la mayoría de las lesiones que generan invaginación gastroduodenal tienen un tamaño mayor a $4 \mathrm{~cm}$. La reducción endoscópica es una opción, sin embargo, la tasa de recurrencia es alta. La resección endoscópica, como reportan Yamauchi, et al., puede ser exitosa en pacientes con alto riesgo quirúrgico ${ }^{3,13,14}$.

\section{Conclusiones}

La invaginación gastroduodenal es una presentación clínica muy infrecuente y su diagnóstico puede ser difícil por sus síntomas inespecíficos. La tomografía computarizada de abdomen es una herramienta valiosa para su estudio.

\section{Financiamiento}

No se ha recibido ningún tipo de financiación para la realización de este artículo.

\section{Conflicto de intereses}

Los autores declaran no tener conflicto de intereses.

\section{Responsabilidades éticas}

Protección de personas y animales. Los autores declaran que para esta investigación no se han realizado experimentos en seres humanos ni en animales.

Confidencialidad de los datos. Los autores declaran que han seguido los protocolos de su centro de trabajo sobre la publicación de datos de pacientes.

\section{Derecho a la privacidad y consentimiento infor-} mado. Los autores han obtenido el consentimiento informado de los pacientes y/o sujetos referidos en el artículo. Este documento obra en poder del autor de correspondencia.

\section{Bibliografía}

1. Al-Radaideh AM, Omari HZ, Bani-Hani KE. Adult intussusception: A 14year retrospective study of clinical assessment and computed tomography diagnosis. Acta Gastroenterol Belg. 2018;81(3):367-72.

2. Ssentongo P, Egan M, Arkorful TE, Dorvlo T, Scott O, Oh JS et al. Adult intussusception due to gastrointestinal stromal tumor: A rare case report, comprehensive literature review, and diagnostic challenges in low-resource countries. Case Rep Surg. 2018;2018:1395230.

3. Zhou Y, Wu XD, Shi Q, Xu CH, Jia J. Gastroduodenal intussusception and pylorus obstruction induced by a c-KIT-negative gastric gastrointestinal stromal tumor: case report and review of the literature. Z Gastroenterol. 2018;56(4):374-9.

4. Yamauchi K, Iwamuro M, Ishii E, Narita M, Hirata N, Okada H. Gastroduodenal Intussusception with a gastric gastrointestinal stromal tumor treated by endoscopic submucosal dissection. Intern Med. 2017;56(12):1515-9.

5. Sorour M, Kassem M, El-Hamid A, El-Riwini M, Nasr A. Gastrointestinal stromal tumors (GIST) related emergencies. Int J Surg. 2014;12(4):269-80.

6. Rittenhouse DW, Lim PW, Shirley LA, Chojnacki KA. Gastroduodenal intussusception of a gastrointestinal stromal tumor (GIST): case report and review of the literature. Surg Laparosc Endosc Percutan Tech. 2013;23(2):e70-3.

7. Wilson MH, Ayoub F, McGreal P, Collins C. Gastrointestinal stromal tumour presenting as gastroduodenal intussusception. BMJ Case Rep. 2012;2012:bcr2012006787.

8. Castro G, Montalvo D, Moyano C, Tapia R, Alarcón R. Invaginación gastroduodenal como presentación de un tumor del estroma gastrointestinal gástrico. Rev Chil Cir. 2016;68(4):316-8.

9. Giménez S, Raichholz G, Froullet C, Dumoulin S, Brouver H, Sañudo J. Hallazgos en TC de las neoplasias de intestino delgado. Revista Argentina de Diagnóstico por Imágenes. 2016;5(15):7-15.

10. Bejarano D, González P, Rodríguez Á, Bolongo R. Tumores del estroma gastrointestinal. Revisión de nuestra experiencia. Rev Chil Cir. 2014;67(4):386-92.

11. Gyedu A, Reich SB, Hoyte-Williams PE. Gastrointestinal stromal tumour presenting acutely as gastroduodenal intussusception. Acta Chir Belg. 2011;111(5):327-8.

12. Jameel ARA, Segamalai D, Murugaiyan G, Shanmugasundaram R, Obla NB. Gastroduodenal intussusception due to gastrointestinal stromal tumour (GIST). J Clin Diagn Res. 2017;11(8):PD09-PD10.

13. Basir N, Yaakub AB, Kafeel G, Telisinghe PU, Tan KK, Sharif F, et al. Gastroduodenal intussusception as a first manifestation of gastric gastrointestinal stromal tumor. Turk J Gastroenterol. 2012;23(2):185-6.

14. Đokić M, Novak J, Petrič M, Ranković B, Štabuc M, Trotovšek B. Case report and literature review: patient with gastroduodenal intussusception due to the gastrointestinal stromal tumor of the lesser curvature of the gastric body. BMC Surg. 2019;19(1):158. 